\title{
STM/AFM OBSERVATIONS OF Co/Cu MAGNETIC MULTILAYERS
}

\author{
M. Aleszkiewicz ${ }^{a}$, R. Kalinowski ${ }^{a}$, R. Czajka ${ }^{b}$, W. Polewska ${ }^{b}$, \\ A. WAWRO ${ }^{a}$, L.T. BACZEWSKI ${ }^{a}$ AND J. RAULUSZKIEWICZ ${ }^{a}$ \\ ${ }^{a}$ Institute of Physics, Polish Academy of Sciences \\ Al. Lotników 32/46, 02-668 Warsaw, Poland \\ ${ }^{b}$ Institute of Physics, Poznan Technical University \\ Piotrowo 3, 60-965 Poznań, Poland
}

UHV deposited magnetic $\mathrm{Co} / \mathrm{Cu}$ multilayers were investigated by means of scanning tunneling microscopy (STM) and atomic force microscopy (AFM). Surface of the sample i.e. upper covering layer in "plane" configuration and individual sublayers in "cross-section" configuration were investigated. A possibility of structure characterization of metallic multilayers by STM and AFM in the cross-section configuration is demonstrated.

PACS numbers: $68.65 .+\mathrm{g}, 61.16 . \mathrm{Ch}, 68.55 . \mathrm{Jk}$

Short-period superlattices are a new class of materials with highly unusual properties, making them potentially useful for future device technologies. Because of extremely high spatial resolution and sensitivity to electronic properties the scanning tunneling microscopy and spectroscopy (STS) are natural techniques to apply to the nanometer-scale structure characterization at interfaces. There is a lot of papers reporting application of STM/STS in the so-called "cross-section" configuration (CS-STM, Fig. 1) for researching semiconducting superstructures $[1,2,3]$. Profiling of individual non-semiconducting sublayers by means of CS-STM technique has not been reported in literature yet. Our CS-STM and CS-AFM measurements of magnetic $\mathrm{Co} / \mathrm{Cu}$ multilayers show the possibility of applying these techniques to metallic structures.

Magnetic Co/Cu multilayers (MML) were deposited in ultrahigh vacuum system $\left(2 \times 10^{-10} \mathrm{Tr}\right)$ on Si substrates, kept at $40-50^{\circ} \mathrm{C}$ during the growth process, covered with $\mathrm{Cu}$ buffer layer. Composition of studied MML is described as follows: $\mathrm{Cu}$ buffer $\mathrm{Q}_{1} / n \times(\mathrm{Co} X / \mathrm{Cu} Y) /$ covering layer $Q_{2}$, where $n$ is the number of $\mathrm{Co} / \mathrm{Cu}$ periods, $Q_{1}, X, Y, Q_{2}$ - thickness of buffer layer, Co and Cu sublayers, covering layer, respectively. $X$ and $Y$ were varied between $2.8 \mathrm{~nm}$ and $11.2 \mathrm{~nm}$. Reflection high-energy electron diffraction (RHEED) analysis performed in situ after growth process revealed polycrystalline structure with appearing texture of individual sublayers. Layered structure was checked by low-angle X-ray reflectometry. Typical diffraction pattern showed several peaks separated by Kiessig fringes. 


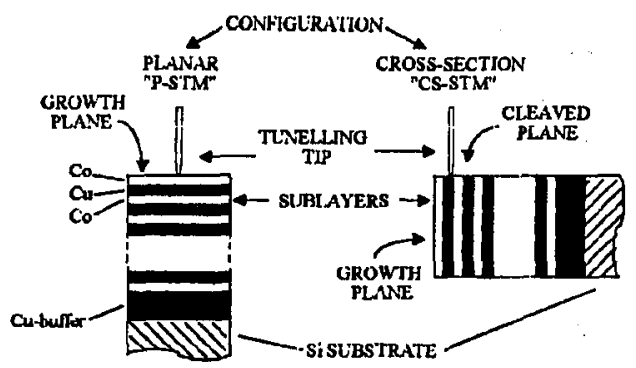

Fig. 1. Schematic of P-STM and CS-STM.

Fig. 2.
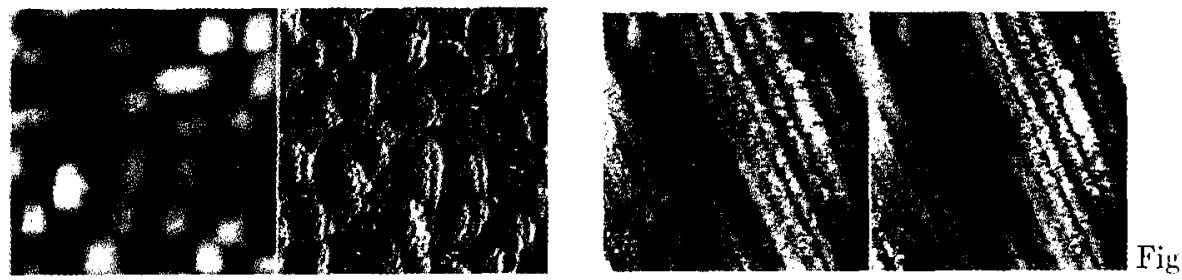

Fig. 2. P-STM $200 \mathrm{~nm} \times 200 \mathrm{~nm}$ image of Co covering layer. Left: topographical image (vertical scale $10 \mathrm{~nm}$ ). Right: current image (vertical scale $35 \mathrm{p} \AA$ ).

Fig. 3 CS-STM $500 \mathrm{~nm} \times 500 \mathrm{~nm}$ topographical images, vertical scale $5 \mathrm{~nm}$. Left: sample scanned from left to right. Right: scanned from right to left.

MML were imaged in both P-STM ("planar"-STM, Fig. 1) and CS-STM configurations using two different STM units and AFM. In "cross-section" techniques fabrication method of a suitable surface is crucial. The most reliable results would be expected after breaking the sample in situ in UHV chamber immediately after deposition of a structure. In our experiment an equivalent method of protecting samples against oxidation is breaking and measuring them in silicon oil. IIere we present results of measurements performed in air on freshly cleaved samples.

As an example we present measurements performed on a sample of composition: $\mathrm{Cu} 42 \mathrm{~nm} / 19 \times$ (Co $11.2 \mathrm{~nm} / \mathrm{Cu} 11.2 \mathrm{~nm}$ )/Co $16.8 \mathrm{~nm}$. P-STM images reveal cluster structure (Fig. 2) of upper Co covering layer. Co clusters are of size of order $30-50 \mathrm{~nm}$ and $5 \mathrm{~nm}$ height and are formed from smaller grains of about few $\mathrm{nm}$ diameter and less than $1 \mathrm{~nm}$ vertical corrugation.

In Fig. 3 a CS-STM image is presented. Individual sublayers are visible as series of grains corresponding to the structure of covering Co layer seen in Fig. 2. Each layer consists of separate grains as thick as presumed thickness of the deposited layer. It is possible to distinguish between neighbouring layers because the grains are aligned parallel to the sample edge. Vertical corrugation of grains in both kinds of layers is less than $1 \mathrm{~nm}$. The same size of grains in covering layer and in all sublayers means that during the deposition process the granular structure is conserved in each subsequent layer in the modulated structure. 


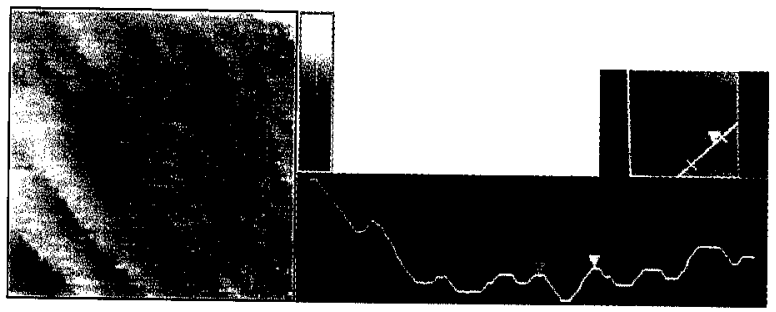

Fig. 4. CS-STM. Left: topographical image $190 \mathrm{~nm} \times 170 \mathrm{~nm}$, vertical scale $31 \mathrm{~nm}$. Right: CS profile; horizontal distance between arrows $19 \mathrm{~nm}$.

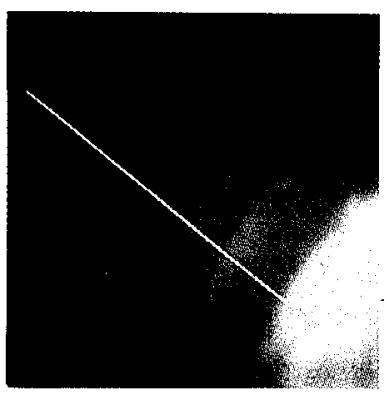

Length:

$450.11 \mathrm{~nm}$

Height:

$27.466 \mathrm{~nm}$

Angle:

38.14

(a)

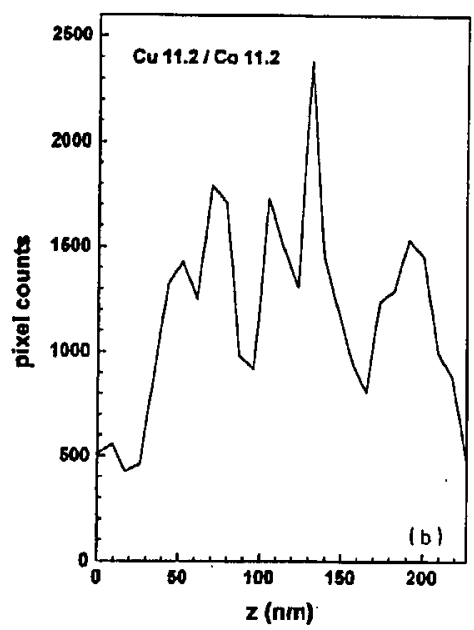

Fig. 5. CS-AFM. Left: topographical image $450 \mathrm{~nm} \times 450 \mathrm{~nm}$, vertical scale $30 \mathrm{~nm}$; below: surface profile along the white line. Right: statistics on topography image distance between peaks corresponds to the step height.

Figure 4 presents another CS-STM image of the same sample. Dimensions of individual sublayers in all presented measurements are consistent with thicknesses defined in the process of deposition.

Figure 5 depicts an AFM topography image and surface profile, measured in contact mode along the white line. Several parallel terraces, typical of layered material, are clearly visible. Their heights correspond to the thickness of individual layers or to its multiplicity which can be seen from statistical calculation presented in the same figure. On this basis we associate these terraces to the individual interfaces of the multilayers.

There are at least three mechanisms, why different metallic sublayers can be distinguished in the tunneling process: (i) different electronic properties, (ii) different thickness of oxides, which can add some topography elements to (i), (iii) to- 
pography introduced by breaking the sublayers according to differences in their elastic properties. Observation of sublayers by AFM suggests the predominance of mechanisms (ii) and (iii). Further experiments are in progress.

It is demonstrated that scanning probe microscopy techniques (STM, AFM) can be used as an effective tool for the characterization of modulated structures in the "cross-section" configuration not only for semiconductor superlattices, but also for metallic multilayers. Individual sublayers and their structure can be visualized and characterized. The improvement of experimental techniques and measurements in situ will result in more precise interface description.

\section{Acknowledgments}

This work is partially supported by the Committee for Scientific Research under grants Nos. 2.P302.007.06 and 8.T11.B05309. The purchase of AFM was sponsored by The Foundation for Polish Science under SEZAM'94 Program, project No. $33 / 94$.

\section{References}

[1] H.W.M. Salemink, O. Albrektsen, J. Vac. Sci. Technol. B 10, 1799 (1992).

[2] H.W.M. Salemink, O. Albrektsen, P. Koenraad, Phys. Rev. B 45, 6946 (1992).

[3] M.B. Johnson, U. Maier, H.-P. Meier, H.W.M. Salemink, Appl. Phys. Lett. 63, 1273 (1993). 